\title{
CONCEPTUAL DECISION MODEL
}

\author{
Miki Sirola \\ Helsinki University of Technology Laboratory of Computer and Information Science \\ P.O.Box 5400 FIN-02015 HUT, Finland, Miki.Sirola@hut.fi, http://www.cis.hut.fi/miki/
}

\begin{abstract}
Decision making is mostly based on decision concepts and decision models built in decision support systems. Type of decision problem determines application. This paper presents a conceptual decision model that utilises rulebased methodologies, numerical algorithms and procedures, statistical methodologies including distributions, and visual support. Selection of used decision concepts is based on case-based needs. Fine tuning of the model is done during construction of the computer application and analysis of the case examples. A kind of decision table is built including pre-filtered decision options and carefully chosen decision attributes. Each attribute is weighted, decision table values are given, and finally total score is calculated. This is done with a many-step procedure including various elements. The computer application is built on G2 platform. The case example choice of career is analysed in detail. The developed prototype should be considered mostly as an advisory tool in decision making. More important than the numerical result of the analysis is to learn about the decision problem. Evaluation expertise is needed in the development process. The model constructed is a kind of completed multi-criteria decision analysis concept. This paper is also an example of using a theoretical methodology in solving a practical problem.
\end{abstract}

Keywords: - Decision Models, Decision Concepts, Decision Support Systems, Modelling

\section{INTRODUCTION}

There are many aspects in decision making that need to be paid special attention to. Various decision concepts have been composed and many kind of decision models have been built to provide the decision support systems with best possible aid. Decision maker has to be able to integrate all valuable information available and ennoble out good enough decision in each particular decision case.

Visual support is often very valuable for the decision maker. Visual support can mean very many things of course. Visualisation of process data is a basic example of giving to the decision maker information that can be valuable. Visualisation of process variables starts from simple plots and time series, and may go into more and more complicated development.

Such support methodologies as decision tables, decision trees, flow diagrams, and rule-based methods should be mentioned. Calculation algorithms e.g. for optimisation are often needed as well. Selection criteria formation and decision option generation are also important parts of the decision process, systematically used for example in multi-criteria decision analysis methodology. Statistical methodologies, distributions, object models, agents and fuzzy models are also sometimes introduced as parts of decision models. Simulation for tracking purposes and prediction should not be forgotten either. In system level applications many of these concepts are needed and utilised further.

The decision types can be divided into long-term decisions and short-term decisions. The number of objectives, possible uncertainty, time dependence, etc. also affect to the decision problem perspective. Sometimes the decisions need to be made on-line and sometimes off-line. All these factors need to be kept in mind when the problem solving methodology is chosen.

Comparing risk and cost is a common methodology in decision making. Choosing the preferences with competing priorities is an important point. Cumulative quality function and chained paired comparisons are examples of more specific methodologies used in decision making. Measures in decision making is also an interesting point. Deterministic decision making has its own measures mostly based on value and utility theory, while stochastic decision making uses statistical measures such as distributions.

Decision concepts have been reviewed in reference [1]. Although decision making is applied in many areas, the literature seems to concentrate on economy and production planning. In used methodologies there exist more variation. For instance decision analysis approach and knowledgebased technologies are commonly used concepts. 
Decision making in handling sequences, resource allocation, network design, sorting problems and classification are examples of problem types studied in detail. Some of these issues are also discussed in references [2], [3] and [4].

In the conceptual decision model presented in this paper only carefully considered features have been used. Some of the earlier presented techniques have been selected into this model. This model utilises rule-based methodologies, numerical algorithms and procedures, statistical methodologies including e.g. distributions, and visual support. Rule-based methodologies are used for instance in preliminary elimination of decision options, algorithms and procedures e.g. in calculation of weight coefficients, and statistical distributions in evaluating the values in a kind of decision table. This utilisation is explained more in detail in the next chapter about the decision concept and model.

The selection of the used decision concepts in the model is based on case-based needs. By analysing real decision problems the most suitable features and methodologies have been taken in use. If some feature is noticed to be unnecessary in the decision process, it has been left out from the final decision model. Also missing features have been completed on the way. The concept of the model is first planned on rough level, and then fine tuned during the examination of the case examples.

The type of the decision problem determines the application. In a decision situation there often exist alternatives. Sometimes the alternatives appear in frequent sets. In each decision situation there is a certain history that can be more or less known. Statistical support, production of solutions, filtering and selection are all needed in certain steps. Situation-based assessment is very important for instance in a case as if checkers. This game located on a draught-board may be analysed by using different area sizes or numbers of elements. The number of possible combinations increases very fast with the size.

Other possible cases worth to mention are e.g. selection of career, buying decision (car is the classical example), or an optimisation of a going route. The selection of career may include such attributes as inclination, interest, economy, risk, etc. The buying decision has such possible attributes as price, technical qualities, colour, model, etc. In this paper the case of choice of career is analysed more in detail. The computer application of this case example has been built with G2 expert system shell.

\section{DECISION CONCEPT OF THE MODEL}

The conceptual decision model is presented here first on a rough level, and then by going more a more into details when getting more into the examined case itself. It was found out that the rulebased methods, algorithms and procedures, statistical methods such as distributions, and visual support are the most suitable methodologies and give the most desired features for the decision model in question. The whole concept in use consists of these elements just mentioned.

In the model a kind of decision table is built including decision options and decision attributes (see Fig. 1). The decision attributes can also be called decision criteria. Each decision attribute has a weight coefficient, and each decision option can be valued in regard to each attribute. This far the table is quite similar that is used in quantitative analysis of multi-criteria decision analysis. In fact also the formula calculating the final numerical result is exactly the same:

$$
v_{t o t}\left(a_{i}\right)=\sum_{i=1}^{n} w_{j} v_{j}\left(a_{i}\right)
$$

where $a_{i}$ is decision alternative $i, w_{j}$ is weight coefficient of criteria $j$ and $v_{j}\left(a_{j}\right)$ is a scaled score of the decision alternative $i$ compared to criterion $j$.

The decision problem shapes during the analysis process. First the decision attributes are defined for the case in question. Then the decision options are created. The decision options are filtered with a rulebased examination, and only the most suitable ones are selected for the final analysis. Similar procedure is possible to realise with the decision attributes as well.

The weight coefficients are calculated with an algorithm based on pairwise comparisons and step by step adjustment through all attributes in the final analysis. This procedure is adjusted for each case separately. The use of statistical distributions comes into the figure when the values in the decision table are given. Some attributes with regard to their corresponding decision option are valued by using information in statistical tables. Historical data is one of the elements used in constructing these tables. This method and its realisation is explained more in detail in the next chapter about the computer application.

The decision table helps also the decision maker to perceive the decision problem visually. It is of course possible to use many kinds of visualisation methodologies in addition, visualisations that help the decision maker to picture the decision problem better than just pure numbers in a decision table. But already the decision problem shaped into a decision table gives great help in understanding the decision problem better. 


\section{COMPUTER APPLICATION}

The computer application is realised in G2 expert system shell. The presented realisation scheme (see Fig. 1) is for the case choice of career, but it can be easily generalised for any of the cases mentioned in this paper. Some key features of the realisation are also documented here.

The decision table itself is realised as a freedom table. The changing values comes either from arrays such as choice of career (options), value of attribute (criteria) and weight of attribute (weight coefficients), or some of the functions explained later on (numerical values in the decision table). The result is calculated with formula (1) as it was mentioned in chapter 2. Also the array values are calculated as outputs of procedures or functions.

An object class career has been defined, and four subclasses inclination, interest, economy and risk. The career candidates such as mathematician, natural scientist, linguist, lawyer, economist and trucker are defined as instances of the object class career. The rule base (not seen in the figure) takes care for instance of the filtering of the initial decision options. Minimum limit values are defined for the weight coefficients of the attributes, and based on these comparisons the final decision options are selected.

As already mentioned there exist procedures and functions calculating the weight coefficients and numerical values in the decision table. The procedures and functions calculating the numerical values in the decision table also utilises accessory tables including information from statistical distributions about the attributes in the decision table. The realisation scheme of the calculation of the weight coefficients and numerical table values is not described in every detail in this paper.

\section{CASE EXAMPLE ABOUT THE CHOICE OF CAREER}

In the case more than thirty persons choice of career is analysed. The selection process is more or less the same with each person, or at least so similar, that only one such example is discussed in detail. No statistical analysis is done for the whole random sample. The qualities of the decision model and the concept used are considered more important, and these features are best lighted through an illustrative example. The decision table for person number 17 is seen in Fig. 1.

For the person number 17 the decision option filtering gives out three options: mathematician, lawyer and trucker. So in the decision table of Fig. 1 the career 1 is mathematician, career 2 is lawyer, and career 3 is trucker. The decision attributes are inclination, interest, economy and risk. Here inclination means the genetic feasibility of the person for such a career. Interest means the subjective willingness to choose the career. Economy means the statistical income of each career type, and risk the statistical possibility of getting unemployed in each profession.

As input for the procedure calculating weight coefficients are given such things as each persons subjective evaluation and a kind of general importance. As already mentioned the attributes economy and risk are valued by using tables including information from statistical distributions, while the other attributes are valued by different means. A subjective measure is used in valuing the attribute interest, while the attribute inclination is valued by combined technology including subjective, statistical and kind of quality measures.

Person number 17 seems to have most inclination for the career of a lawyer, and most interest for the career of mathematician. The statistics shows best income for the career of lawyer, and smallest risk for the career of trucker. Note that the attribute risk is in inverse scale, so a big number means a small risk (which is considered to be a good quality), while all other attributes are in normal scale (big number means big inclination, interest or economy). The scale of the values in the decision table is from 0 (worst possible) to 5 (best possible).

The numerical result shows clearly that person number 17 should choose the option lawyer. The second best choice would be mathematician, and clearly last comes trucker. This result is so clear that sensitivity analysis is not needed with this person. In many cases the sensitivity analysis shows the week points of the analysis by making clear how different parameters affect to the final result. It must be noted though that the attribute risk was given very small weight. Still many people consider this attribute rather important in this context. In this case the inclination has been given rather high emphasis. The importance of attributes economy and interest are located somewhere between.

This tool should be considered as some kind of advisory tool, and by no means as an absolute reference for the final choice. Although with the person number 17 two of the attributes clearly point to the choice of lawyer career, there are still two important attributes that disagree with this opinion. For instance many people think that one should follow the voice of interest in such things as choosing a career. Still this kind of analysis can be very informative for the decision maker to find out the other motives for such choices that may often be more hidden. 


\section{DISCUSSION}

Conceptual decision model has been built by first collecting the desired decision concept by combining in a new way existing decision support methodology. Then the decision model has been built by iterating the details while the computer application is being built and the case examples analysed. The computer application is programmed on G2 platform. The case example of choice of career is analysed more in detail. Also other case examples have been used in the development of the computer application, and also in the fine tuning of the concept and model itself.

The developed prototype should be considered mostly as an advisory tool in decision making. The features have been selected with thorough care, and therefore the desired qualities are mostly found from the final application. Also the realisation puts up some restrictions in the possibilities of course, but in general I think that we can be rather satisfied with the results achieved.

The decision model includes rule-based methodologies, numerical algorithms and procedures, statistical methodologies such as distributions, and visual support. The use of these methodologies in the model has been explained more in detail in previous chapters. The role of visual support is not very remarkable in this application, although it can be considered generally quite important and also an essential part of the concept.

There exist many possibilities in making errors, for instance in calculating the weight coefficients and in producing the values in the decision table, but mostly the sensitivity analysis helps to find out the week points in the analysis. Plausible comparison of different attributes is also a problem. By giving the weight coefficients it is tried to put the attributes in a kind of order of importance, but to justify the comparison itself is often problematic. For instance comparison of risk and cost is not always correct by a common opinion.

The numerical result of the analysis is not the most important result achieved. The most important result is to find out what are the most important decisive factors in the whole decision making process, and therefore to learn more about the problem itself. Already the better understanding of the problem helps in finding out a good solution, even if the decision maker does not agree with the numerical result given by the tool. The decisive factors and their order of importance are found from the decision attributes (criteria) of the formulated decision problem.

Sometimes the revelation of hidden motives helps the decision maker. This often happens during the long procedure of playing with the decision problem, from the beginning of the problem formulation to the final analysis and even documentation. Better understanding by learning during the decision problem is one of the key issues that this paper is trying to present.

Evaluation expertise is also very important to include in the solution procedure of the decision problem. Otherwise we are just playing with random numbers without a real connection to the decision itself that we are tying to solve.

This paper is an example of using a theoretical methodology in solving a practical problem. The model constructed is a kind of completed multicriteria decision analysis (MCDA) concept. The decision table itself is very similar, but the difference is in the way that different components and values of the table are produced. In ordinary MCDA method more handwork is done, while this system is rather far automated.

The case about choice of career is a typical one time decision. The case of buying a new car is very similar in this regard. On the other hand the game checkers is completely different decision problem type. In that case rather similar decision situations come out repeatedly.

The role of historical data, retrieval and prediction becomes more important. The case of optimising a going route is again different, third type of problem. Only the first one of these problems was analysed in this paper. The others were just very shortly introduced.

The realisation platform for the computer application is G2 expert system shell. This environment is quite suitable for this kind of purpose. G2 is very strong in heuristics, and not so good in numerical calculation. Although both them are needed in this application, the need of very heavy numerical calculation is not so essential. As a kind of combinatorial methodology has been developed, also other platforms could be considered. G2 was just a natural choice to make a first prototype to test the ideas presented in this paper.

Although many persons were analysed in the choice of career case example experiment, no statistical analysis for the whole random sample was made. To concentrate more in this issue is one clear future need. As a tool capable of handling rather large amounts of data in a moderate time has been built, a natural way to proceed is to widen the scope into this direction.

This expert system concept can be used in various decision situations that people meet in everyday life. This kind of decision table related to an expert system is also a good choice to make decision process understandable for human being. 
I owe an acknowledgement for the Laboratory of Automation Technology of our university for the possibility of using G2 expert system shell as a platform of the computer application, which made the analysis of the case examples possible.

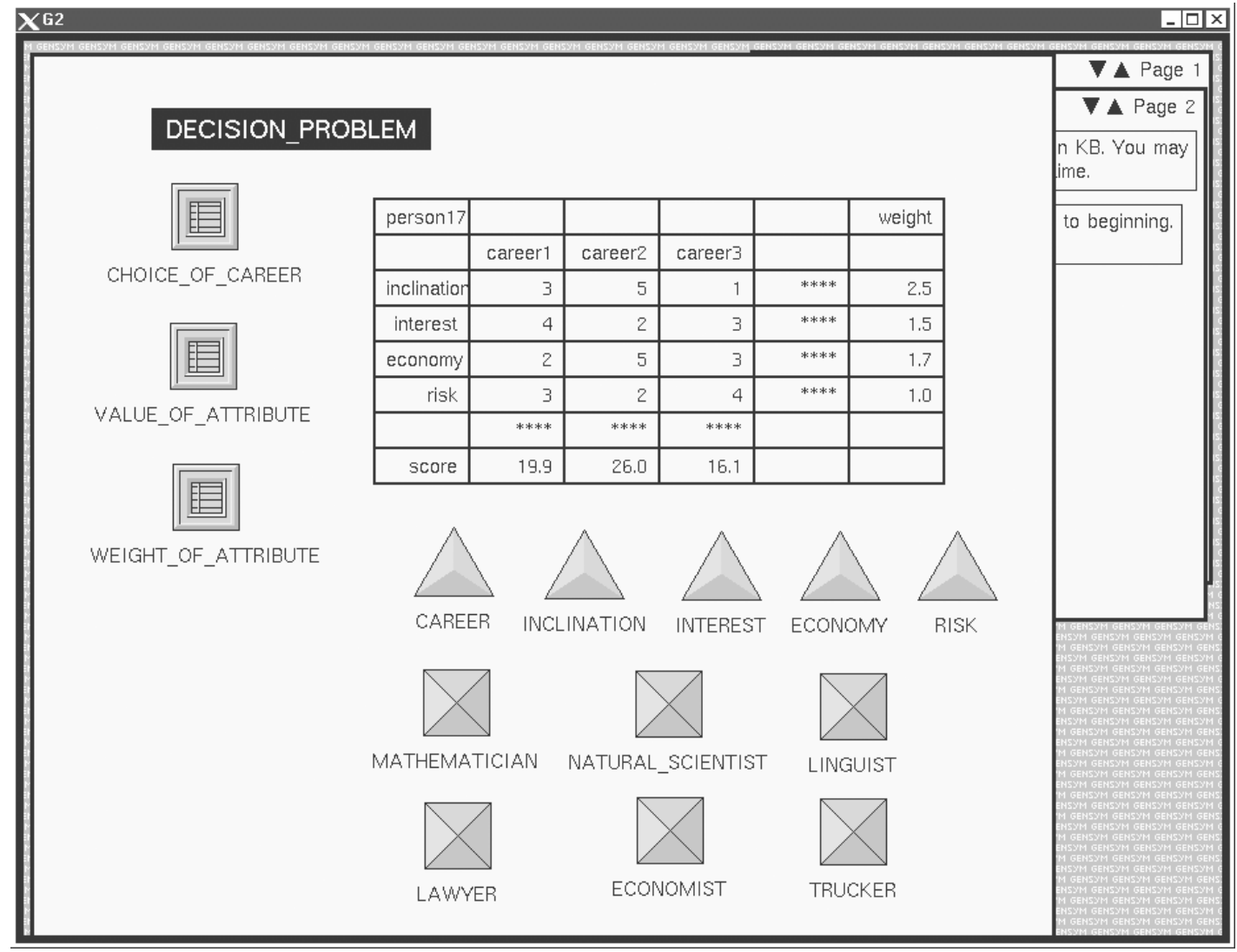

Fig. 1 - A window of the G2 application where the case choice of career is analysed for person number 17.

\section{REFERENCES}

[1]M. Sirola. Decision concepts. Proceedings of IEEE Second International Workshop on Intelligent Data Acquisition and Advanced Computing Systems: Technology and Applications, Lviv, Ukraine, 2003.

[2]D. Ashmos, D. Duchon, R. McDaniel. Participation in strategic decision making: the role of organizational predispositions and issue interpretation. Decision sciences. Atlanta, 1998.

[3]R. Santhanam, J. Elam. A survey of knowledgebased systems research in decision sciences, The Journal of the Operational Research Society. Oxford (1998), pp. 1980-1995.

[4]J. Rummel. An empirical investigation of costs in batching decisions. Decision sciences. Atlanta, 2000.

\begin{abstract}
Miki Sirola has been a Laboratory Engineer in the Laboratory of Computer and Information Science in Helsinki University of Technology since 1998. He received MSc 1988 in Electrical Engineering (System Control and Automation) from Helsinki University of Technology, LicTech 1993 in Electrical Engineering (Automation) and DTech 1999 in Automation and System Technology (Automation) from the same university. Prior to Helsinki University of Technology, he worked at VTT (Technical Research Centre of Finland) Automation as research scientist (1987-1998) and at Institutt for Energiteknikk (OECD Halden Reactor Project) as research scientist (1992-1993). His research interests are in knowledge-based systems and decision support.
\end{abstract}

